\title{
Methicillin-resistant Staphylococcus aureus nasal colonization in a department of pediatrics: a cross-sectional study
}

\author{
Francesco Gesualdo ${ }^{1 *}$, Manuela Onori ${ }^{1}$, Dafne Bongiorno ${ }^{2}$, Floriana Campanile ${ }^{2}$, Emanuela Carloni ${ }^{1}$, \\ Livia Mancinelli ${ }^{1}$, Cristina Russo ${ }^{1}$, Alberto Villani ${ }^{1}$, Diletta Valentini ${ }^{1}$, Massimiliano Raponi ${ }^{1}$, Alberto E Tozzi ${ }^{1}$ \\ and Stefania Stefani ${ }^{2}$
}

\begin{abstract}
Background: We describe methicillin-resistant Staphylococcus aureus (MRSA) nasal carriage at admission in patients admitted to a Department of Pediatrics.

Methods: All patients received a nasal swab at admission. A questionnaire was administered and molecular genetics analyses were performed on all identified MRSA isolates.

Results: We enrolled 785 patients, affected with both acute and chronic diseases. MRSA nasal colonization prevalence was 1.15\% (Cl: 0.5607\%-2.093\%). Methicillin-sensitive Staphylococcus aureus (MSSA) nasal colonization prevalence at admission was 19.75\% (Cl 17.07\%-22.64\%). Only one MRSA isolate carried the SCCmec V variant; all other isolates carried the SCCmeclV variant. Five out of 9 MRSA-colonized patients had an underlying condition. Antibiotic therapy in the previous 6 months was a protective factor for both MRSA (OR 0,66; 95\% Cl: 0,46-0,96) and MSSA (OR 0,65; 95\% Cl: 0,45-0,97) colonization. A tendency to statistical significance was seen in the association between hospitalization in the 6 months prior to admission and MRSA colonization at admission (OR 4,92; 95\% Cl: 0,97-24,83). No patient was diagnosed with an S. aureus infection during hospitalization.
\end{abstract}

Conclusions: The majority of our MRSA colonizing isolates have community origins. Nevertheless, most MRSA-colonized patients had been hospitalized previously, suggesting that strains that circulate in the community also circulate in hospital settings. Further studies should elucidate the role of children with frequent contact with health care institutions in the circulation of antibiotic resistant strains between the hospital and the community.

Keywords: Methicillin-resistant Staphylococcus aureus, Nasal carriage, Risk factors

\section{Background}

In the last decade, after an increasing trend in the incidence of methicillin-resistant Staphylococcus aureus (MRSA) infections in Europe and the United States, both in adults and in children [1,2], encouraging data on a general reduction of invasive MRSA infections have been recently reported [3,4]. The proportion of $S$. aureus isolates identified as methicillin-resistant is stabilizing or decreasing in most European countries [5]. Nevertheless, MRSA remains a public health priority as the proportion of MRSA of all

\footnotetext{
* Correspondence: f.gesualdo@gmail.com

'Bambino Gesù Children's Hospital IRCCS, Rome, Italy

Full list of author information is available at the end of the article
}

S. aureus isolates is still above $25 \%$ in 8 out of 28 countries, mainly in Southern and Eastern Europe, including Italy [5].

Reports on MRSA nasal colonization in children have shown variable prevalence figures, ranging from 0 to over $20 \%$, with European figures of $3.3 \%$ or lower [6]. Most of the surveys that investigated nasal carriage have been conducted on healthy pediatric populations, or on patients admitted to Intensive Care Units [7]. Only few studies have investigated nasal carriage in General Pediatric Wards [8].

MRSA nasal carriers may be at greater risk of developing an MRSA infection compared to methicillin-sensitive S. aureus (MSSA) carriers and to non-carriers, in particular in intensive care unit (ICU) settings $[9,10]$. Therefore, programs for preventing MRSA nosocomial transmission

\section{Ciomed Central}

(c) 2014 Gesualdo et al.; licensee BioMed Central Ltd. This is an open access article distributed under the terms of the Creative Commons Attribution License (http://creativecommons.org/licenses/by/2.0), which permits unrestricted use, distribution, and reproduction in any medium, provided the original work is properly cited. 
have been implemented, including screening of highrisk patients, isolation of colonized individuals and decolonization $[11,12]$. However, a comprehensive evaluation of prevention strategies is still lacking, and some strategies may have an unexpected impact on colonized children and their families, possibly leading to a feeling of social stigma [13].

The objectives of the present study were to assess the prevalence of MSSA and MRSA nasal colonization among patients admitted to a pediatric department, to describe molecular genetic characteristics of the colonizing isolates, and to identify epidemiological risk factors associated with MSSA and MRSA nasal colonization.

\section{Methods}

\section{Setting}

The Bambino Gesù Children's Hospital, located in Rome, Italy, is a 607-bed comprehensive medical centre for pediatric healthcare and research. The Department of Pediatrics includes 132 beds in one single building divided into 4 floors by level of assistance.

\section{Study design and patient recruitment}

Over a 3-month period between February 8, 2010 and May 14, 2010 we conducted a cross-sectional study enrolling patients admitted to 3 wards belonging to the Department of Pediatrics. Children admitted to the wards were affected by general pediatric acute or chronic illnesses, rheumatologic diseases, infectious diseases (excluding HIV), bronchopneumopathies, cystic fibrosis, endocrinological diseases and genetic diseases.

No age or pathology constraints were applied in the selection of the population. Patients not speaking Italian, English, Spanish and French were excluded. Written, informed consent was obtained from patients older than 18 years of age, or from parents of patients younger than 18 years of age, together with patient assent, or written, informed consent if patient was older than 12 years.

Enrolled patients underwent an anterior nasal swab in one of the nares within $12 \mathrm{~h}$ of admission. A questionnaire was administered to the parents or to the patient to collect demographic and epidemiological data, including: birth date, sex, parents' data (age, level of education and employment), number of siblings, number of household members, number of bedrooms, presence of smoking people at home, presence of domestic animals, presence of an household member working in a healthcare facility, current breastfeeding (for children $<2$ years), daycare center or school attendance, extra-school sport activity (for children $>4$ years), nail biting (for children $>4$ years), diagnosis of a chronic underlying disease, past diagnosis of pneumonia or of an invasive infection, emergency room visit during last 6 months, hospital admission during last 6 months, administration of antibiotic therapy during last 6 months, upper respiratory tract infection during last week. The study was approved by the Bambino Gesù Children's Hospital Ethical Committee.

\section{Laboratory methods for S. aureus identification}

Samples were collected from anterior nares using the ESwab (Copan, Brescia, Italy) collection and transport device, composed of a flocked swab adsorbed into $1.0 \mathrm{ml}$ of Amies liquid transport medium. For each nasal sample two aliquots $(10 \mu \mathrm{l}$ each) of ESwab liquid medium were streaked onto mannitol salt agar, Chapman (bioMérieux, Marcy l'Etoile, France) and on chromogenic agar MRSASelect (Bio-Rad, Nazareth Eke, Belgium) respectively, and incubated at $37^{\circ} \mathrm{C}$ for $18-24 \mathrm{~h}$. Colonies grown on Chapman agar having morphology suggestive of $S$. aureus, or mauve in color presumptively MRSA positive on chromogenic agar were subcultured onto 5\% sheep blood agar. Phenotypical identification was assessed by Vitek2 system (bioMérieux Marcy l'Etoile, France) according to the manufacturer's instructions.

In vitro susceptibility testing for oxacillin, erythromycin, clindamycin, gentamicin, ciprofloxacin, rifampicin, trimethoprim-sulfamethoxazole, mupirocin, vancomycin, teicoplanin (Sigma Chemical, St. Louis, MO, USA) and linezolid (Pfizer, Groton, CT, USA), was further performed by the broth microdilution method to determine the minimum inhibitory concentrations (MICs), following the CLSI guidelines. The EUCAST guidelines were also used for comparison $[14,15]$. In particular the oxacillin susceptibility was interpreted according to CLSI recommendations, and in cases of discrepancy susceptibility testing was repeated.

The penicillin-binding protein ( $\mathrm{PBP} 2 \mathrm{a})$ latex agglutination test (SLIDEX MRSA Detection, bioMérieux Marcy l'Etoile, France) was used as an additional phenotypic confirmatory test for presumptively detected MRSA strains.

\section{PCR and SCCmec typing}

Genomic DNA was extracted from all bacterial cultures and used as a template for amplification.

All isolates were screened for the presence of the mecA, lukS-PV/lukF-PV genes and ACME-locus. PCR experiments were carried out following procedures previously reported [16-18]. The SCCmec (StaphylococcalChromosomal-Cassette $\mathrm{mec}$ ) types were first determined by a multiplex-PCR protocol previously described, and assigned to the corresponding types [19]. Moreover, for the characterization of the SCCmec IV subtype an alternative multiplex-PCR protocol was performed [20], the results were confirmed with other different multiplex-PCR protocols, focusing on the mec gene complex and the $c \mathrm{cr}$ gene complex [21] and hsdRMS operon [16].

The sequences of the seven housekeeping genes used for MLST, corresponding to the allelic profile arcC- 
aroE-glp-gmk-pta-tpi-yqiL, were obtained by comparing the sequences obtained for all MRSA strains in study with those in the MLST database http://saureus.mlst.net [22]. All sequence-types (STs) described in the study were deposited on the MLST website. PCR products were analyzed by electrophoresis on $1 \%$ or $2 \%$ agarose gels (Sigma Aldrich, Saint Louis Mo, USA).

\section{Statistical methods}

Sample size calculation was based on the assumption of a carriage rate of $2 \%$. Around 785 subjects needed to be screened, in order to estimate the carriage rate with adequate precision (95\% confidence intervals between 1\% and $3 \%$ for 785 subjects).

Proportions of the social-demographic characteristics of each group of colonized/not-colonized children were compared through $\chi^{2}$ or Fisher exact test where appropriate. Statistical tests were considered significant at a pvalue $<0.05$ level.

In order to study the association between potential risk factors and colonization status at the time of hospital admission, we performed a univariate analysis through a logistic regression model in which odds ratios and 95\% CI were calculated. Potential risk factors included in the analysis as independent variables were all those investigated in the questionnaire, plus age at admission.

In each model colonization status was included as a dependent variable, as follows: colonized patients vs non-colonized; MSSA colonized vs non-colonized patients and MRSA colonized vs non-colonized patients. At the univariate level, the analysis was restricted to children aged $\leq 4$ years for "sport"; $\geq 3$ years for "nail biting"; $<2$ years for "breastfeeding".

Variables with a p-value $<0.20$ at the univariate analysis were selected to be included in the multivariate analysis using a logistic regression model, with nasal carriage being the dependent variable as reported above. Since none of the variables analyzed on a restricted population at the univariate level had a $\mathrm{p}$ value $<0.20$, the multivariate model included the entire population. Statistical software STATA 11 was used for data analysis.

\section{Results}

\section{Study population}

We enrolled 785 patients from 3 pediatric wards of the Department. Mean age of patients was 4.62 years (median: 2.66, range: 0.03-27.7), of which 403 (51\%) were males. Other characteristics of the population are reported in Table 1. Among the enrolled patients, no one had been admitted for a symptomatic $S$. aureus infection or was diagnosed with a nosocomial $S$. aureus infection during hospitalization.

\section{Colonization prevalence and risk factors}

At admission, a total of 164 patients (20.89\%; 95\% CI: $18.16 \%-23.84 \%)$ were colonized by S. aureus; 155 patients (19.75\%; 95\% CI: 17.07\%-22.64\%) were colonized by MSSA and 9 patients (1.15\%; 95\% CI: $0.5607 \%-$ $2.093 \%)$ were colonized by MRSA.

Among the MRSA colonized patients, 5 had an underlying condition (mucopolysaccharidosis, arthrogryposis, neurofibromatosis, type 1 diabetes, osteoid osteoma). The remaining 4 patients had been admitted with the following diagnoses: bronchiolitis, rickettsiosis, chickenpox, sepsis. Six of the MRSA-colonized patients had already been hospitalized in the last 6 months. Combining information on underlying diseases and previous hospitalizations, 3 out of 9 MRSA colonized patients did not have risk factors for previous healthcare exposures.

Risk factors for MSSA and MRSA carriage identified by multivariate analysis are reported in Table 2 .

According to the multivariate analysis, compared to non-colonized patients, MRSA colonized patients were more likely to have been hospitalized in the previous 6 months. Antibiotic therapy in the last 6 months was found as a protective factor for MRSA colonization. The multivariate analysis did not identify age as a risk factor for MRSA colonization, while MSSA-colonized patients were more likely to belong to the 6-13 age group compared to non-colonized children.

\section{MRSA antimicrobial susceptibility and molecular characteristics}

Antimicrobial susceptibility and molecular characteristics of the isolates are reported in Table 3.

Nine MRSA were isolated, all characterized by phenotypic and molecular methods, as described in the Methods section.

All isolates were oxacillin resistant, 4/9 were erythromycin resistant, 3/9 ciprofloxacin and rifampicin resistant, 1/9 gentamicin resistant, and only one isolate was resistant to cotrimoxazole. All strains were susceptible to clindamycin, mupirocin, linezolid and glycopeptides.

MLST analysis showed 7 different STs associated with different variants of SCCmec IV and only one with SCCmec V.

Only 1 strain carried $l u k S-P V / l u k F-P V$ genes, and did not harbour the arginine catabolic mobile element (ACME), associated toUSA300 clone.

\section{Discussion}

This study assessed MRSA nasal colonization in a population of children admitted to a General Pediatric Department, including a large group of patients affected by chronic diseases. Our results show an MSSA nasal colonization prevalence of $19.75 \%$ and an MRSA nasal colonization prevalence of $1.15 \%$. 
Table 1 General characteristics of patients included in the study

\begin{tabular}{|c|c|c|c|c|c|c|c|c|c|}
\hline \multirow{2}{*}{ Number of patients; $N$} & & \multicolumn{2}{|c|}{ MSSA } & \multicolumn{2}{|c|}{ MRSA } & \multicolumn{2}{|c|}{ Not colonized } & \multicolumn{2}{|c|}{$\begin{array}{c}\text { Total } \\
\text { population }\end{array}$} \\
\hline & & 155 & & 9 & & 621 & & 785 & \\
\hline Male; N, \% & & 87 & 56.1 & 4 & 44.4 & 312 & 50.2 & 403 & 51.3 \\
\hline \multirow[t]{3}{*}{ Age; N, \% } & $0-5$ & 95 & 61.3 & 8 & 88.9 & 459 & 73.9 & 562 & 71.6 \\
\hline & $6-13$ & 52 & 33.5 & 1 & 11.1 & 121 & 19.5 & 174 & 22.2 \\
\hline & $>=14$ & 8 & 5.2 & 0 & 0.0 & 41 & 6.6 & 49 & 6.2 \\
\hline \multirow[t]{4}{*}{ Parent nationality; N, \% } & Italian & 138 & 89.0 & 7 & 77.8 & 554 & 89.2 & 699 & 89.0 \\
\hline & European & 6 & 3.9 & 1 & 11.1 & 27 & 4.3 & 34 & 4.3 \\
\hline & Extra-European ${ }^{1}$ & 8 & 5.2 & 1 & 11.1 & 27 & 4.3 & 36 & 4.6 \\
\hline & Mixed & 3 & 1.9 & 0 & 0.0 & 14 & 2.3 & 16 & 2.0 \\
\hline Mother's age, years; mean, SD & & 36.5 & 7.1 & 32.9 & 5.8 & 35.8 & 6.7 & 35.9 & 6.8 \\
\hline Father's age, years; mean, SD & & 38.8 & 7.3 & 37.7 & 6.0 & 39.0 & 7.1 & 38.9 & 7.1 \\
\hline Mothers with high school diploma; N, \% & & 111 & 71.6 & 4 & 44.4 & 464 & 74.7 & 579 & 73.8 \\
\hline Fathers with high school diploma; N, \% & & 96 & 61.9 & 6 & 66.7 & 412 & 66.3 & 514 & 65.5 \\
\hline Unemployed mothers; N, \% & & 62 & 40.0 & 5 & 55.6 & 230 & 37.0 & 297 & 37.8 \\
\hline Unemployed fathers; N, \% & & 11 & 7.1 & 0 & 0.0 & 18 & 2.9 & 29 & 3.7 \\
\hline Presence of underlying illness; N, \% & & 58 & 37.4 & 4 & 44.4 & 160 & 25.8 & 222 & 28.3 \\
\hline Hospitalization length; mean, SD & & 6.8 & 6.8 & 7.6 & 7.5 & 7.0 & 6.0 & 7 & 6.2 \\
\hline
\end{tabular}

${ }^{1}$ Among the 36 extra-european patients, 20 (56\%) originated from Asian countries, 9 (25\%) from African countries, 7 (19\%) from South American countries. The only extra-european MRSA colonized patient was from Vietnam.

MRSA nasal colonization in the pediatric age has been investigated by a number of studies, which showed variable prevalence figures and diverse risk factors [7,8,23-27]. Most studies investigated carriage in a healthy pediatric population $[7,25,27]$. A smaller number of studies focused on Intensive Care Units $[28,29]$, or on patients with underlying diseases [24]. Few studies investigated colonization in a general pediatric ward and our study gives new insights on this topic. Two studies [30,31] investigated MRSA nasal carriage in different hospital departments, including a pediatric ward, and in both studies no MRSA was identified. In a multicenter study carried out in children's hospitals in Switzerland, one MRSA was identified in 1,350 investigated patients (prevalence: 0.07\%) [8].

We molecularly characterized the 9 MRSA isolates and demonstrated the presence of different strains with community or nosocomial origins.

A circulation of the ST8-CAMRSA-IV.a clone in Italy has been already demonstrated in patients affected by invasive infections (necrotizing pneumonia, skin and soft tissue infections, sepsis), always in association with PVL production. Among the colonizing isolates belonging to the ST8-CAMRSA-IV.a clone, only one carried the lukS-PV/lukF-PV genes [32].

Table 2 Multivariate risk factors for S. aureus, MSSA and MRSA colonization

\begin{tabular}{|c|c|c|c|c|c|c|c|c|c|c|c|}
\hline & & Risk factor & & & SA vs & $s$ nega & tive & & RSA v & is nega & tive \\
\hline & $\begin{array}{c}\text { Total N. of patients } \\
\text { with risk factor (n. 785) }\end{array}$ & $\begin{array}{c}\text { N. of patients MSSA } \\
\text { with risk factor (n. 155) }\end{array}$ & $\begin{array}{c}\text { N. of MRSA-colonized } \\
\text { patients with risk factor (n. 09) }\end{array}$ & $\overline{O R}$ & $95 \%$ & $\% \mathrm{Cl}$ & $p$ & OR & & $\% \mathrm{Cl}$ & $p$ \\
\hline Age 6-13 & 174 & 52 & 1 & 1,77 & 1,04 & 2,98 & 0,034 & & & NS & \\
\hline $\begin{array}{l}\text { Unemployed } \\
\text { father }\end{array}$ & 29 & 11 & 0 & 2,67 & 1,19 & 5,99 & 0,017 & & & NS & \\
\hline Chronic illness & 222 & 58 & 4 & 1,56 & 1,01 & 2,39 & 0,043 & & & NS & \\
\hline $\begin{array}{l}\text { Antibiotics last } \\
6 \text { months }\end{array}$ & 509 & 92 & 4 & 0,65 & 0,45 & 0,97 & 0,034 & 0,66 & 0,46 & 0,96 & 0,033 \\
\hline $\begin{array}{l}\text { Hospital } \\
\text { admission last } \\
6 \text { months }\end{array}$ & 319 & 63 & 6 & & & NS & & 4,92 & 0,97 & 24,83 & 0,053 \\
\hline
\end{tabular}


Table 3 Patient's diagnosis, molecular characteristics and antibiotic resistance of MRSA colonizing isolates

\begin{tabular}{llccccccccccccccccc}
\hline \multicolumn{1}{l}{ Diagnosis } & ST & SCCmec & mec-complex & ccr-complex & PVL & OXA & E & DA & CN & CIP & RD & SXT & VA & TEC & LNZ & MUP \\
\hline 1 & Mucopolysaccharidosis & 8 & IV.C & B & A2B2 & - & R & R & S & S & R & R & R & 0,5 & 0,25 & 2 & 1 \\
2 & Chickenpox & 8 & IV.a & B & A2B2 & - & R & S & S & S & R & S & S & 1 & 0,25 & 2 & 0,25 \\
3 & Bronchiolitis & 8 & IV.a & B & A2B2 & + & R & S & S & S & S & S & S & 0,5 & 0,5 & 2 & 1 \\
4 & Arthrogryposis & 22 & IV.c & B & A2B2 & - & R & S & S & S & S & S & S & 1 & 0,25 & 2 & 0,5 \\
5 & Neurofibromatosis 1 & 45 & IV.a & B & A2B2 & - & R & R & S & S & S & R & S & 0,5 & 1 & 2 & 4 \\
6 & Type 1 diabetes & 15 & IV.a & B & neg & - & R & S & S & S & R & S & S & 0,5 & 0,25 & 2 & 2 \\
7 & Osteoid osteoma & 5 & IV.a & B & A2B2 & - & R & R & S & S & S & S & S & 1 & 0,25 & 4 & 0,25 \\
8 & Rickettsiosis & 1 & IV.a & B & A2B2 & - & R & R & S & R & S & R & S & 0,5 & 0,25 & 2 & 1 \\
9 & Sepsis & 30 & V & C & C & - & R & S & S & S & S & S & S & 0,5 & 0,25 & 2 & 2 \\
\hline
\end{tabular}

$O X A$, oxacillin; $E$, erythromycin; $D A$, clindamycin; $C N$, gentamicin; $C I P$, ciprofloxacin; $R D$, rifampicin; $S X T$, trimethoprim-sulfamethoxazole; $V A$, vancomycin; $T E C$, teicoplanin; $L N Z$, linezolid; $M U P$, mupirocin. $R=$ resistant; $S=$ sensitive.

The ST1-CAMRSA-IV.a, already described as USA400 in the United States, circulates worldwide in different countries [33], and in Italy was recently isolated from pigs (nasal samples and dust samples collected in holdings of breeding and production pigs) [34].

The ST30 clones, with different pulsotype and genetic characteristics, have been reported in many areas of the world. This clone was previously described in Italy in a patient with necrotizing pneumonia, and associated with SCCmec IV and the lukS-PV/lukF-PV genes [32]. On the contrary, our isolate carried for the first time SCCmec V and $c c r C$ type recombinases, and no lukS-PV/lukF-P genes. This variability in acquiring diverse genetic elements (SCCmec and PVL-encoding phages) suggested that this clone, in Italy, has evolved in multiple occasions.

Circulation of the ST45-CAMRSA-IV.a has been recently demonstrated in Cambodian children [35]. Interestingly, our ST45-CAMRSA-IV.a was isolated from a recently adopted Vietnamese child.

ST22-CAMRSA-IV was previously described as an epidemic infective clone in adults (EMRSA 15) [36] and has been recently isolated on Portuguese public buses [37]. $S$. aureus belonging to ST15 have rarely been reported as methicillin-resistant. One colonizing ST15-MRSA was reported from Africa and ST15 was previously associated with community-onset MSSA bacteremia among adults in an Italian study $[38,39]$. The present study identified one of the MRSA colonizing isolates showing ST15-SCCmec type IV.

The only ST5 SCCmec IV colonized a child with previous hospitalizations for an osteid osteoma.

We observed a tendency to statistical significance in the association between MRSA colonization and hospitalization in the 6 months prior to the nasal sampling, confirming the results reported by other authors [40,41]. Nevertheless, the majority of our MRSA isolates have community origins. This observation shows that strains that circulate in the community also circulate in hospital settings. To this regard, more research is needed to investigate the role of children that have frequent contacts with health care institution (eg. children affected with underlying disease and chronic conditions) as potential MRSA vectors between the hospital and the community [6].

According to our findings, use of antibiotics in the 6 months before admission protects from both MSSA and MRSA carriage. Other authors have previously reported a protective effect of antibiotic therapy in the last 6 months on MSSA colonization [25,26].

In the complex interaction of factors affecting emergence and spread of multridrug-resistant organisms, antimicrobials are known to exert a selective pressure on resistant clones. We found, instead, a protective effect of antibiotics administered in the previous 6 months on MRSA colonization. In order to elucidate this result, more data regarding prescriptions (kind of infection, class of prescribed antibiotics, days of therapy) would be needed. Although, this kind of information is easily subject to recall bias, therefore we decided not to include it in our questionnaire. A cohort study with a prospective design would be needed for appropriately characterizing the effect of antibiotic use on resistant clones.

Our study has some limitations. First, parents not speaking Italian, English, Spanish and French were not enrolled in the study. This might have led to an underestimation of the MRSA carriage prevalence. However, as foreign patients are a very limited proportion of the total hospitalized patients, the effect of this selection bias could be negligible.

We did not explore colonization in other body sites (throat, perineum), which could have led to a misclassification of colonized patients as non-colonized [42]. Another possible limitation is the low number of patients that were positive to MRSA for which risk factors were evaluated. Thus, associations found in our study may be not sufficient to explain the outcome (nasal carriage).

\section{Conclusion}

Our findings show that strains that circulate in the community also circulate in hospital settings: in fact, the 
majority of our MRSA colonizing isolates have community origins; nevertheless, most MRSA-colonized patients had been hospitalized previously. More research is needed to elucidate the role of antibiotic prescriptions in the emergence of MRSA clones, and to analyze the possible role of children with frequent contact with health care centers (eg. children affected with chronic diseases) as MRSA vectors between the hospital and the community.

\section{Competing interests}

The authors declare that they have no competing interests.

\section{Authors' contributions}

FG coordinated the study and drafted the manuscript, MO and LM performed the microbiological analyses, DB and FC performed the molecular genetic analyses, EC performed the statistical analysis, DV coordinated patient enrollment, CR and AV participated in drafting the manuscript, MR revised the manuscript, AET and SS supervised the study, revised the manuscript and gave final approval of the version to be published. All authors read and approved the final manuscript.

\section{Acknowledgements}

This work was supported by the Italian Ministry of Health (contract no. 200601X002321) and the Regione Sicilia (contract no. 201101X002657).

\section{Author details}

'Bambino Gesù Children's Hospital IRCCS, Rome, Italy. ${ }^{2}$ Department of Bio-Medical Sciences, Section of Microbiology, University of Catania, Catania, Italy.

Received: 4 September 2013 Accepted: 17 December 2013 Published: 10 January 2014

\section{References}

1. Klevens RM, Morrison MA, Nadle J, Petit S, Gershman K, Ray S, Harrison LH, Lynfield R, Dumyati G, Townes JM, Craig AS, Zell ER, Fosheim GE, McDougal LK, Carey RB, Fridkin SK: Active Bacterial Core surveillance (ABCs) MRSA Investigators: invasive methicillin-resistant Staphylococcus aureus infections in the United States. JAMA 2007, 298(15):1763-1771.

2. Johnson AP, Pearson A, Duckworth G: Surveillance and epidemiology of MRSA bacteraemia in the UK. J Antimicrob Chemother 2005, 56(3):455-462.

3. Johnson AP, Sharland M, Goodall CM, Blackburn R, Kearns AM, Gilbert R, Lamagni TL, Charlett A, Ganner M, Hill R: Enhanced surveillance of methicillin-resistant Staphylococcus aureus (MRSA) bacteraemia in children in the UK and Ireland. Arch Dis Child 2010, 95(10):781-785.

4. Kallen AJ, Mu Y, Bulens S, Reingold A, Petit S, Gershman K, Ray SM, Harrison LH, Lynfield R, Dumyati G: Health care-associated invasive MRSA infections, 2005-2008. JAMA 2010, 304(6):641.

5. European Centre for Disease Prevention and Control (ECDC): Antimicrobial resistance surveillance in Europe 2010. Annual report of the European antimicrobial resistance surveillance network (EARS-net). 2010: [http:// ecdc.europa.eu/en/publications/Publications/1111_SUR_AMR_data.pdf].

6. Gesualdo F, Bongiorno D, Rizzo C, Bella A, Menichella D, Stefani S, Tozzi AE: MRSA nasal colonization in children: prevalence meta-analysis, review of risk factors and molecular genetics. Pediatr Infect Dis J 2013, 32(5):479-485.

7. Fluegge K, Adams B, Luetke Volksbeck U, Serr A, Henneke P, Berner R: Low prevalence of methicillin-resistant staphylococcus aureus (MRSA) in a southwestern region of Germany. Eur J Pediatr 2006, 165(10):688-690.

8. Heininger U, Datta F, Gervaix A, Schaad UB, Berger C, Vaudaux B, Aebi C, Hitzler M, Kind C, Gnehm HE, Frei R, PIGS/MRSA Study Group: Prevalence of nasal colonization with methicillin-resistant Staphylococcus aureus (MRSA) in children a multicenter cross-sectional study. Pediatr Infect Dis J 2007, 26(6):544-546.

9. Honda H, Krauss MJ, Coopersmith CM, Kollef MH, Richmond AM, Fraser VJ, Warren DK: Staphylococcus aureus nasal colonization and subsequent infection in intensive care unit patients: does methicillin resistance matter? Infect Control Hosp Epidemiol 2010, 31(6):584-591

10. Milstone AM, Goldner BW, Ross T, Shepard JW, Carroll KC, Perl TM: Methicillin-resistant Staphylococcus aureus colonization and risk of subsequent infection in critically ill children: importance of preventing nosocomial methicillin-resistant Staphylococcus aureus transmission. Clin Infect Dis 2011, 53(9):853-859.

11. Muto CA, Jernigan JA, Ostrowsky BE, Richet HM, Jarvis WR, Boyce JM, Farr BM SHEA: SHEA guideline for preventing nosocomial transmission of multidrug-resistant strains of Staphylococcus aureus and Enterococcus. Infect Control Hosp Epidemiol 2003, 24(5):362-386.

12. Edgeworth JD: Has decolonization played a central role in the decline in UK methicillin-resistant Staphylococcus aureus transmission? A focus on evidence from intensive care. J Antimicrob Chemother 2011, 66(Suppl 2):ii41-ii47.

13. Sengupta A, Rand C, Perl TM, Milstone AM: Knowledge, awareness, and attitudes regarding methicillin-resistant Staphylococcus aureus among caregivers of hospitalized children. J Pediatr 2011, 158(3):416-421.

14. Clinical and Laboratory Standard Institutes: Performance standards for antimicrobial susceptibility testing: approved standard M100-S21. 2011

15. European Committe on Antimicrobial Susceptibility Testing: Clinical breakpoints. [http://www.eucast.org/clinical_breakpoints/.

16. Campanile F, Bongiorno D, Borbone S, Venditti M, Giannella M, Franchi C, Stefani S: Characterization of a variant of the SCCmec element in a bloodstream isolate of Staphylococcus intermedius. Microb Drug Resist 2007, 13(1):7-10.

17. Ellington MJ, Yearwood L, Ganner M, East C, Kearns AM: Distribution of the ACME-arcA gene among methicillin-resistant Staphylococcus aureus from England and Wales. J Antimicrob Chemother 2008, 61(1):73-77.

18. Stefani S, Bongiorno D, Cafiso V, Campanile F, Crapis M, Cristini F, Sartor A, Scarparo C, Spina D, Viale P: Pathotype and susceptibility profile of a community-acquired methicillin-resistant Staphylococcus aureus strain responsible for a case of severe pneumonia. Diagn Microbiol Infect Dis 2009, 63(1):100-104.

19. Oliveira DC, de Lencastre $H$ : Multiplex PCR strategy for rapid identification of structural types and variants of the mec element in methicillinresistant staphylococcus aureus. Antimicrob Agents Chemother 2002, 46(7):2155-2161.

20. Milheirico C, Oliveira DC, de Lencastre H: Multiplex PCR strategy for subtyping the staphylococcal cassette chromosome mec type IV in methicillin-resistant Staphylococcus aureus: 'SCCmec IV multiplex'. J Antimicrob Chemother 2007, 60(1):42-48.

21. Kondo $Y$, Ito T, Ma XX, Watanabe S, Kreiswirth BN, Etienne J, Hiramatsu K Combination of multiplex PCRs for staphylococcal cassette chromosome mec type assignment: rapid identification system for mec, ccr, and major differences in junkyard regions. Antimicrob Agents Chemother 2007, 51(1):264-274

22. Cassone M, Campanile F, Pantosti A, Venditti M, Stefani S: Identification of a variant "rome clone" of methicillin-resistant Staphylococcus aureus with decreased susceptibility to vancomycin, responsible for an outbreak in an intensive care unit. Microb Drug Resist 2004, 10(1):43-49.

23. Ridder-Schaphorn S, Ratjen F, Dubbers A, Haberle J, Falk S, Kuster P, Schuster A, Mellies U, Lowe B, Reintjes R, Peters G, Kahl BC: Nasal Staphylococcus aureus carriage is not a risk factor for lower-airway infection in young cystic fibrosis patients. J Clin Microbiol 2007, 45(9):2979-2984.

24. Tramper-Stranders GA, van der Ent CK, Gerritsen SA, Fleer A, Kimpen JL, Wolfs TF: Macrolide-resistant Staphylococcus aureus colonization in cystic fibrosis patients: is there transmission to household contacts? J Antimicrob Chemother 2007, 60(3):665-668.

25. Sdougkos G, Chini V, Papanastasiou DA, Christodoulou G, Stamatakis E, Vris A, Christodoulidi I, Protopapadakis G, Spiliopoulou I: Community-associated Staphylococcus aureus infections and nasal carriage among children: molecular microbial data and clinical characteristics. Clin Microbiol Infect 2008, 14(11):995-1001.

26. Faden H, Lesse AJ, Trask J, Hill JA, Hess DJ, Dryja D, Lee YH: Importance of colonization site in the current epidemic of staphylococcal skin abscesses. Pediatrics 2010, 125(3):e618-e624.

27. Tavares DA, Sa-Leao R, Miragaia M, de Lencastre H: Large screening of CA-MRSA among Staphylococcus aureus colonizing healthy young children living in two areas (urban and rural) of Portugal. BMC Infect Dis 2010, 10:110.

28. Gregory ML, Eichenwald EC, Puopolo KM: Seven-year experience with a surveillance program to reduce methicillin-resistant Staphylococcus aureus colonization in a neonatal intensive care unit. Pediatrics 2009, 123(5):e790-e796. 
29. Kim YH, Chang SS, Kim YS, Kim EA, Yun SC, Kim KS, Pi SY: Clinical outcomes in methicillin-resistant Staphylococcus aureus-colonized neonates in the neonatal intensive care unit. Neonatology 2007, 91(4):241-7.

30. Erdenizmenli M, Yapar N, Senger SS, Ozdemir S, Yuce A: Investigation of colonization with methicillin-resistant and methicillin-susceptible Staphylococcus aureus in an outpatient population in Turkey. Jpn J Infect Dis 2004, 57(4):172-175.

31. Aires De Sousa M, Santos Sanches I, Ferro ML, De Lencastre $H$ : Epidemiological study of staphylococcal colonization and cross-infection in two West African hospitals. Microb Drug Resist 2000, 6(2):133-141.

32. Sanchini A, Campanile F, Monaco M, Cafiso V, Rasigade JP, Laurent F, Etienne J, Stefani S, Pantosti A: DNA microarray-based characterisation of Panton-Valentine leukocidin-positive community-acquired methicillinresistant Staphylococcus aureus from Italy. Eur J Clin Microbiol Infect Dis 2011, 30(11):1399-1408.

33. David MZ, Daum RS: Community-associated methicillin-resistant Staphylococcus aureus: epidemiology and clinical consequences of an emerging epidemic. Clin Microbiol Rev 2010, 23(3):616-687.

34. Franco A, Hasman $H$, lurescia M, Lorenzetti $R$, Stegger M, Pantosti A, Feltrin $F$, lanzano A, Porrero MC, Liapi M, Battisti A: Molecular characterization of spa type $\mathrm{t} 127$, sequence type 1 methicillin-resistant Staphylococcus aureus from pigs. J Antimicrob Chemother 2011, 66(6):1231-1235.

35. Nickerson EK, Wuthiekanun V, Kumar V, Amornchai P, Wongdeethai $N$, Chheng K, Chantratita N, Putchhat H, Thaipadungpanit J, Day NP, Peacock SJ: Emergence of community-associated methicillin-resistant Staphylococcus aureus carriage in children in Cambodia. Am J Trop Med Hyg 2011, 84(2):313-317.

36. Wyllie D, Paul J, Crook D: Waves of trouble: MRSA strain dynamics and assessment of the impact of infection control. J Antimicrob Chemother 2011, 66(12):2685-2688.

37. Simoes RR, Aires-de-Sousa M, Conceicao T, Antunes F, da Costa PM, de Lencastre $\mathrm{H}$ : High prevalence of EMRSA-15 in Portuguese public buses: a worrisome finding. PLoS One 2011, 6(3):e17630.

38. Schaumburg F, Ngoa UA, Kosters K, Kock R, Adegnika AA, Kremsner PG, Lell B, Peters G, Mellmann A, Becker K: Virulence factors and genotypes of Staphylococcus aureus from infection and carriage in Gabon. Clin Microbiol Infect 2011, 17(10):1507-13.

39. Campanile F, Bongiorno D, Falcone M, Vailati F, Pasticci MB, Perez M, Raglio A, Rumpianesi F, Scuderi C, Suter F, Venditti M, Venturelli C, Ravasio V, Codeluppi M, Stefani S: Changing Italian nosocomial-community trends and heteroresistance in Staphylococcus aureus from bacteremia and endocarditis. Eur J Clin Microbiol Infect Dis 2012, 31(5):739-745.

40. Shopsin B, Mathema B, Martinez J, Ha E, Campo ML, Fierman A, Krasinski K, Kornblum J, Alcabes P, Waddington M, Riehman M, Kreiswirth BN: Prevalence of methicillin-resistant and methicillin-susceptible Staphylococcus aureus in the community. J Infect Dis 2000, 182(1):359-362.

41. Milstone AM, Carroll KC, Ross T, Shangraw KA, Perl TM: Communityassociated methicillin-resistant Staphylococcus aureus strains in pediatric intensive care unit. Emerg Infect Dis 2010, 16(4):647-655.

42. Bitterman Y, Laor A, Itzhaki S, Weber G: Characterization of the best anatomical sites in screening for methicillin-resistant Staphylococcus aureus colonization. Eur J Clin Microbiol Infect Dis 2010, 29(4):391-397.

\section{doi:10.1186/1824-7288-40-3}

Cite this article as: Gesualdo et al:: Methicillin-resistant Staphylococcus aureus nasal colonization in a department of pediatrics: a crosssectional study. Italian Journal of Pediatrics 2014 40:3.

\section{Submit your next manuscript to BioMed Central and take full advantage of:}

- Convenient online submission

- Thorough peer review

- No space constraints or color figure charges

- Immediate publication on acceptance

- Inclusion in PubMed, CAS, Scopus and Google Scholar

- Research which is freely available for redistribution

Submit your manuscript at www.biomedcentral.com/submit
Ciomed Central 